\title{
Research of Processes in the Inter-Electrode Intermediation at Electroerosion Processing of Aluminum and Titanium Alloys
}

\author{
Sarilov M Yu* and Reshetnik UE \\ Komsomolsk-on-Amur State University, Russia \\ *Corresponding author: Sarilov M Yu, Komsomolsk-on-Amur State University, Russia
}

\section{ARTICLE INFO}

Received: 幽 September 05, 2019

Published: 幽 September 17, 2019

Citation: Sarilov M Yu, Reshetnik UE. Research of Processes in the Inter-Electrode Intermediation at Electroerosion Processing of Aluminum and Titanium Alloys. Biomed J Sci \& Tech Res 21(3)-2019. BJSTR. MS.ID.003597.

\begin{abstract}
The article presents a study of the processes of electric discharge machining in the interelectrode gap. Peculiarities of electric discharge machining of aluminium and titanium alloys are considered. A hypothesis has been put forward about the possible occurrence of oxide formations (whiskers) during the processing of aluminium and titanium alloys and their influence on the processing. Experimental studies have proved the possibility of the formation of filamentary formations during electrical discharge machining of aluminium alloys and their influence on the accuracy and efficiency of processing. For titanium alloys, the hypothesis has not been confirmed.
\end{abstract}

Keywords: Electric discharge machining; Electric breakdown; Aluminium alloys; Titanium alloys; Interelectrode gap; Pulse frequency; Duty cycle; Oxide mustache; Electrode tool; Electrode blank

\section{Introduction}

Partial or complete destruction of the surface under the influence of external influence is called erosion. Under the electrical erosion of conductive materials refers to the destruction of the surface of the material under the influence of pulses of electric current. The process of electrical discharge machining (EEO) is the destruction of a metal or other conductive material as a result of local exposure to short-term electrical discharges between two electrodes, one of which is a workpiece and the other is an electrode-tool [1]. Electroerosive processing is used in many industries: machine tools, shipbuilding, helicopters, aircraft manufacturing, etc. However, this type of processing is most widely used today in the high technology areas of machine building - engine manufacturing and advanced aircraft building. The constant growth of one of the main indicators of the engine quality level - specific thrust, is ensured, inter alia, through the use of increasingly delicate, slightly rigid spatially complex structural elements, the processing of which is difficult to cut, and in some cases impossible. [2] The details of aircraft have surfaces located in hard-to-reach places that can only be processed using EEE operations. The productivity in this method will increase due to the regulation of processing parameters, that is, the supply of pulses between the electrodes with a high frequency, at the same value of the pulse energy. Because of this, the processing will improve its quality due to the influence of a reduced distance between the electrodes while reducing energy for the alloys we have selected.

\section{Hypothesis}

Based on theoretical and experimental studies, it was suggested that, due to the high chemical (oxidative) activity, high thermal conductivity, low heat capacity and refractoriness when treating aluminium with an electro erosive method, there may be oxide formations (whiskers) obtained from metal, evaporated, molten material, ejected from the hole electrode blanks. These mustaches are aluminium oxide - conductive material, which means that the actual value of the interelectrode gap is reduced. Thus, the proportion of working pulses increases under the same processing conditions, which in turn increases the intensity of the 
erosion process with a simultaneous increase in the temperature of the electrodes. Also, this hypothesis was hypothesized for titanium alloys due to the fact that titanium has close values of chemical activity (especially at high temperatures, which occur when the conductive channel is destroyed) and high thermal conductivity. The hypothesis can be transferred to titanium alloys with assumptions, since the heat capacity and refractoriness of titanium are much higher than aluminium.

The presence of a similar feature in the processing of aluminium and titanium alloys is indirectly confirmed by the results of previous work, since the temperature during the processing of these types of materials is unreasonably high compared to the processing of various types of steel. In turn, changing the processing parameters taking into account this phenomenon will allow us to process materials based on aluminium and titanium more efficiently, that is, to increase productivity by supplying an optimum amount of energy to the interelectrode gap, which will not lead to a deterioration in processing accuracy and an increase in roughness

\section{Main Part}

Conducting an analysis of the materials used in the construction of products used in the aircraft industry, it can be said that titanium and aluminium alloys in all their diversity are prevailing. Light and durable, they become indispensable materials for critical parts, which are subject to the most stringent requirements, both in the quality of the material itself and in the quality of their processing. AND of course, the question arises of increasing the efficiency of processing these types of materials with EEE operations. In a study published in [3], thermal phenomena were studied as an indicator of the intensity of processes occurring in the interelectrode space. Figure 1 shows the changes in the temperature of the workpiece during processing from changes in the main parameters of the processing process (duty cycle and pulse rate).

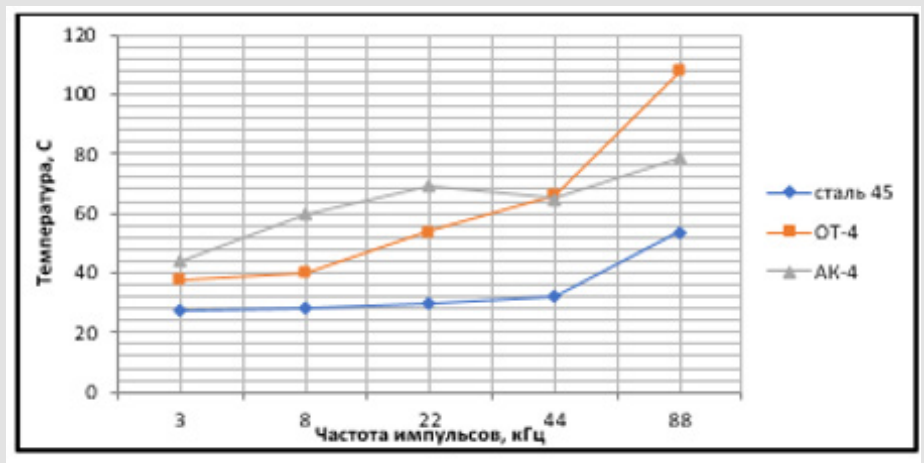

A

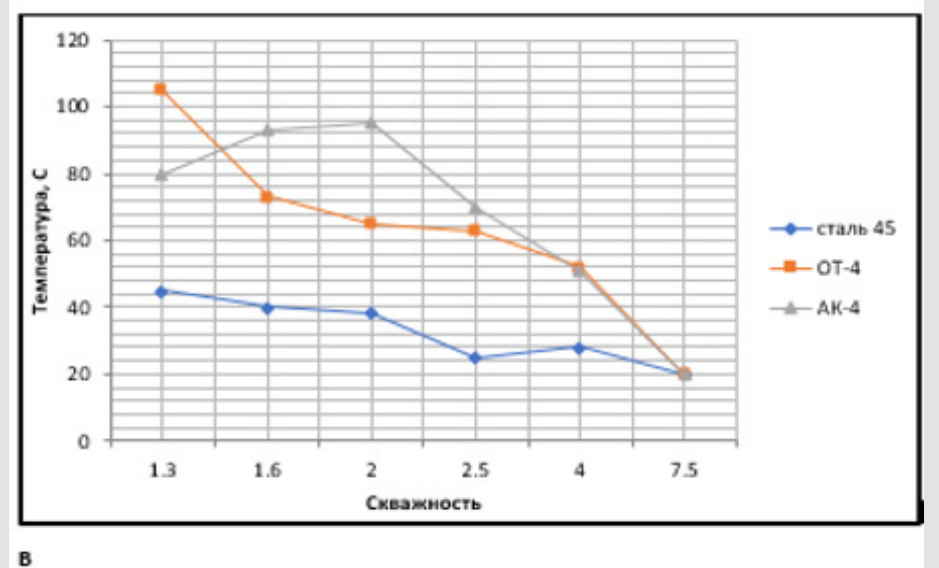

Figure 1: The dependence of temperature on change: pulse frequencies and b) duty cycle.

As you can see from their graphs, the situation with the concentration of thermal energy in an aluminum alloy is similar to that of a titanium alloy, and even a little more in an hour. This is a very paradoxical phenomenon in view of the fact that the thermal conductivity coefficient of aluminum is an order of magnitude greater than that of titanium and almost 3 times greater than that of steel. To compare the thermal effects on these materials, Table 1 shows some constants obtained both experimentally and taken from reference books [2] (Table 1). 
Table 1: Thermophysical characteristics.

\begin{tabular}{|c|c|c|c|c|c|c|c|}
\hline \multirow[b]{2}{*}{ Metal } & \multicolumn{7}{|c|}{ Physical quantity } \\
\hline & $\mathbf{T}_{-}$пл ${ }^{0} \mathrm{C}$ & Т_исп, ${ }^{\circ} \mathrm{C}$ & $\begin{array}{c}\text { q пл, } \\
\text { J/g }\end{array}$ & qисп, J/g & $\lambda, \mathbf{W} / \mathbf{c m} \cdot \mathbf{K}$ & $\mathbf{c}^{*}, \mathbf{J} / \mathbf{g K}$ & $\rho, g / \mathrm{cm}^{3}$ \\
\hline $\mathrm{Al}$ & 660 & $2450 \ldots 2494$ & $396 \ldots 400$ & $10850 \ldots 10900$ & $2,09 \ldots 2,20$ & $0,90 \ldots 0,94$ & 2,7 \\
\hline $\mathrm{Fe}$ & 1535 & $2735 \ldots 2750$ & $247 \ldots 277$ & $6265 \ldots 6340$ & 0,75 & 0,46 & 7,87 \\
\hline $\mathrm{Ti}$ & 16601668 & 32603287 & $\begin{array}{l}358 \\
392\end{array}$ & $8970 \ldots 9227$ & $0,19 \ldots 0,22$ & $0,52 \ldots 0,54$ & 4,5 \\
\hline
\end{tabular}

As can be seen from the table, the aluminium alloy has a very high specific heat coefficient, which indicates the amount of thermal energy that needs to be reported to one gram (kilogram) in order for it to change its temperature by one degree Celsius (Kelvin). All this indicates that the breakdown process has on aluminium and its alloys has its own characteristics that cause the concentration of thermal energy in the processing zone. It is logical to assume that this fact is caused by the influence of the electrode material on the breakdown process, since other conditions of the processing process remain constant for all considered cases. The most probable cause of this anomaly is the distortion of the field in the gap by "whiskers" formed from the oxide film during its destruction during the discharge. The appearance of these oxide whiskers is justified by the high chemical activity of the material itself and taking into account the high temperature of the molten metal in the zone of the hole. The process of the formation of a whisker from aluminium oxide takes an extremely short time, which fits into the temporary picture of the entire breakdown. The expected period of the formation of this phenomenon occurs during the relaxation of the interelectrode gap (MEP), which takes a fairly long period of time by the standards of the duration of the discharge itself, of the order of 10-2 s.

In support of this theory, the fact that the specific heat of evaporation of aluminium is 1.7 times greater than that of iron (steel) also confirms this. That is, when the same amount of energy is supplied to the MEP in one pulse (say, $1 \mathrm{~J}$ ), a large fraction of molten material and a smaller amount of vapor will appear in the hole on the surface of aluminium than in the hole on the surface of the alloy, which will be based on iron. This means that when the gas bubble formed during the discharge collapses and the electrode material is ejected into the MEP, aluminium in the liquid phase will cool faster due to its high specific thermal conductivity and will have time to create an oxide formation. Titanium has a similar oxidizing ability, however, due to the large specific heat coefficient and low heat transfer coefficient, the amount of heated, reactive titanium is smaller and the size of the oxide mustache is smaller. Accordingly, the probability of oxide formation is much less than that of the breakdown on an aluminium alloy.

The existence of the very possibility of such an effect was proposed and considered in [4]. The author of the study investigated numerous effects during breakdown in liquid dielectrics, including those used in EEE. As a result of observation of breakdown cases at various parameters in the study, it was concluded that the formation of oxide formations suggests the presence of chemically active metal, such as a liquid dielectric, the distance between the electrodes, the diameter of the channel, the high intensity of the applied external field when creating a breakdown, a sufficiently high pulse energy realized in the MEP and others. Under these conditions, an oxide whisker takes place, and for remote electrodes and high field strengths, they can reach from tenths and whole millimetres.
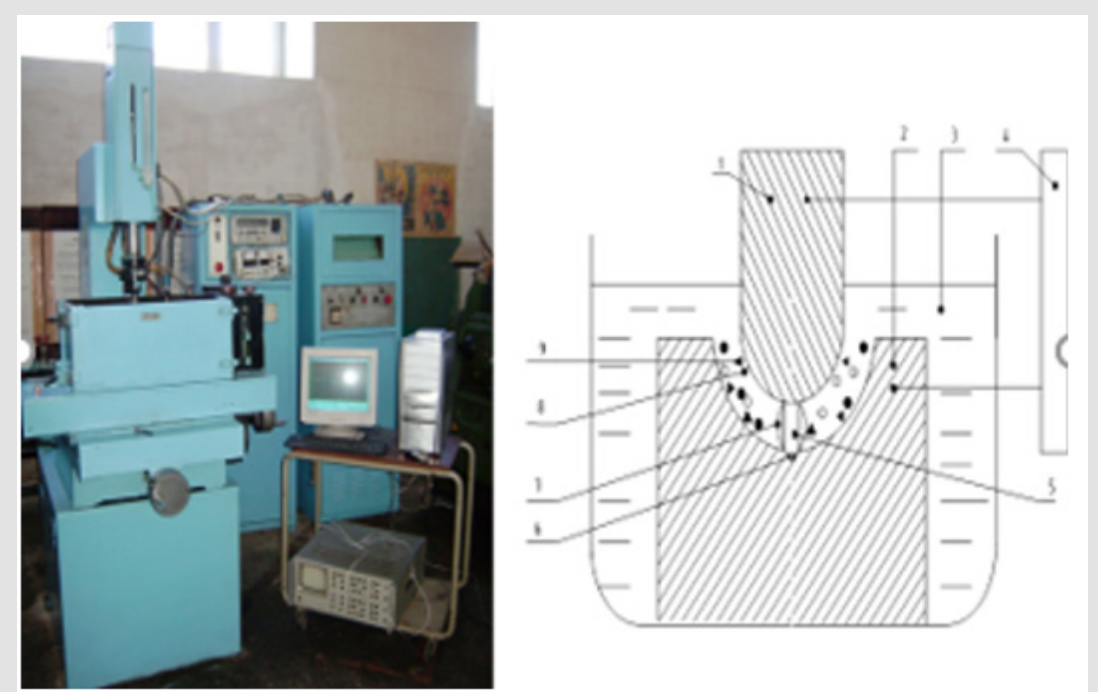

Figure 2: Scheme of the experimental setup 1 - EI; 2 - EZ; 3 - RJ; 4 - generator; 5 - channel charge; 6 - hole formation discharge; 7 - a gas bubble; 8 - metal particles (sludge); 9 - pyrolysis products. 
Analysis of the state of the electrodes during the measurements shows that subsequent discharges on aluminium and titanium electrodes are carried out, as a rule, from the edges of the crater formed from the first breakdown. The formation of craters on the electrodes from other materials does not cause the formation of "whiskers" and almost every subsequent breakdown occurs from a new area of the electrode surface. This phenomenon was observed in all experiments under conditions of a uniform or weakly inhomogeneous field. With the help of experiments it was revealed that from the first breakdown crater edges are formed, and discharges are carried out on aluminium electrodes with the subsequent formation of "whiskers". To confirm this assumption, we conducted experimental studies on a copy-stitching EDM machine with adaptive control model 4L721F1 with a pulse generator ShGI 40-440M (Figure 2). An electrode made of copper M1 was used as the material of the electrode. And as the processed materials, all are the same OT-4 titanium alloy and AK-4 aluminium alloy, as in [3].

As a result of the study, several patterns were obtained. First, with an increase in the pulse energy, the increase in heat release on the surface of the aluminium alloy proceeds at a faster pace, while an almost linear dependence was observed in the considered range of pulse energies in the case of a steel billet electrode. For a titanium alloy, an advance in temperature over an increase in pulse energy was observed only at the very end of the power range available on the machine. This conclusion almost completely fits into the proposed theory. Secondly, the pulse repetition rate, as a rule, does not have a pronounced effect on the concentration of thermal phenomena on the electrodes. However, at any pulse repetition rate, the temperature on the aluminium and titanium electrodes was always higher during processing than on steel. Presumably, the pulse repetition rate does not fundamentally affect the formation of oxide whiskers, since the process of their formation is much less than the time available between the discharges on equipment.

Further investigation of the described phenomenon is possible by recording what is happening in the interelectrode space using a high-resolution high-resolution camera (up to 100,000 frames / sec). Single pulse studies were also carried out using the DGT-EP-03 desktop precision EDM copy-and-flash complex (Figure 3a), as well as the Neophot 2 microscope with a maximum magnification of x1000, shown in Figure 3b.
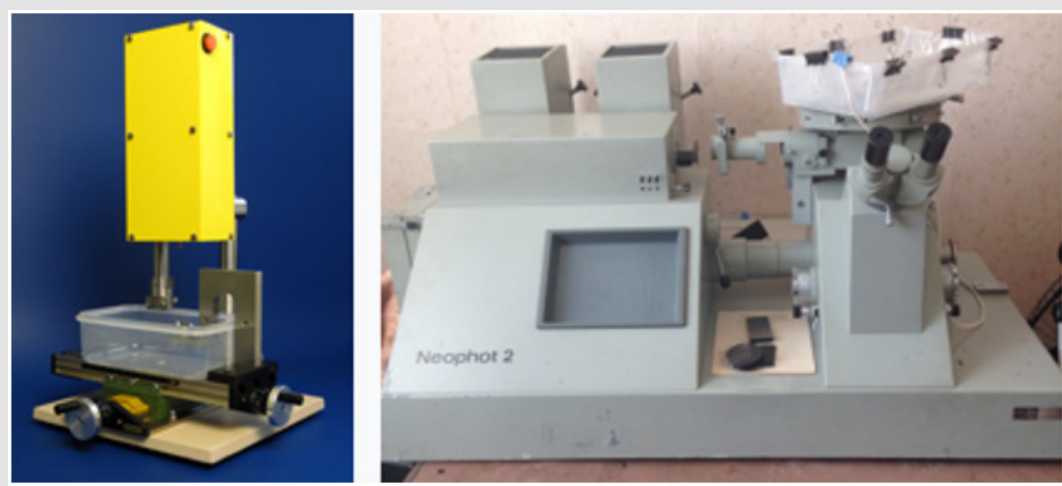

Figure 3: a) DHT-EP-03; b) Neophot 2.

This type of study made it possible to document the presence of oxide whiskers and the conditions for their occurrence and development. It was found that the expansion and narrowing of the channel depends on the introduction of voltage, as well as the time interval, that is, when the introduction is stopped, the expansion of the channel decreases noticeably, and after a lapse of time it narrows due to pressure from the fluid that surrounds the channel. When happens metal cooling due to high ductility and chemical activity, small particles of the material, especially aluminium, are oxidized and melted into an oxide mustache [4].

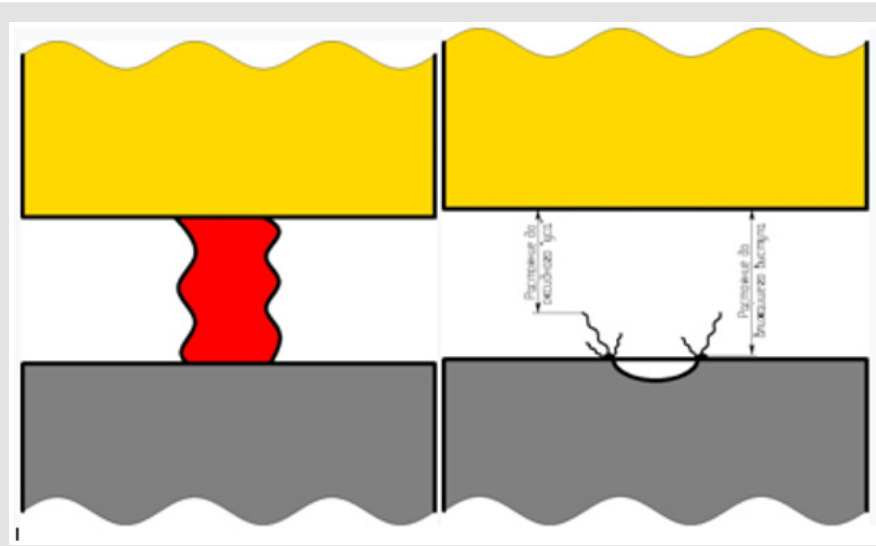

Figure 4: Simplified representation of breakdown on an aluminum alloy. 
The representation of the process is shown in Figure 4. According to approximate miscalculations, the length of these whiskers can reach several millimetres. As a result of studies of the characteristics of aluminium and titanium, we can conclude that the breakdown process of these alloys is similar. Of course, these alloys have differences, since with such parameters of titanium as increased specific heat of fusion and reduced thermal conductivity, a large surface of the material cannot be heated therefore, the oxide "mustache" will not grow. It was found that a slight change in the interelectrode gap can significantly affect the processing process and the final result. The calculation of thermal conductivity confirms that the processing of the materials we have chosen does not quite fit into the theory presented above. Then a hypothesis was put forward about some of the features that accompany the EEE process of materials. Presumably the possible formation

\section{Results and Discussion}

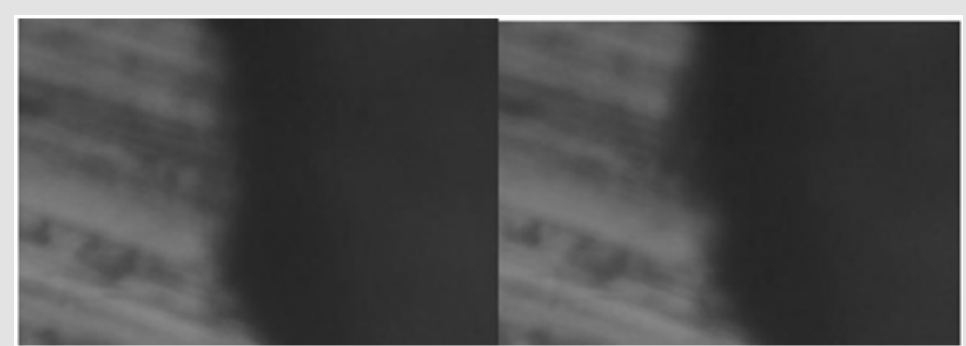

Figure 5: The passage of a pulse between copper EI and aluminum EZ (1000x magnification).

c
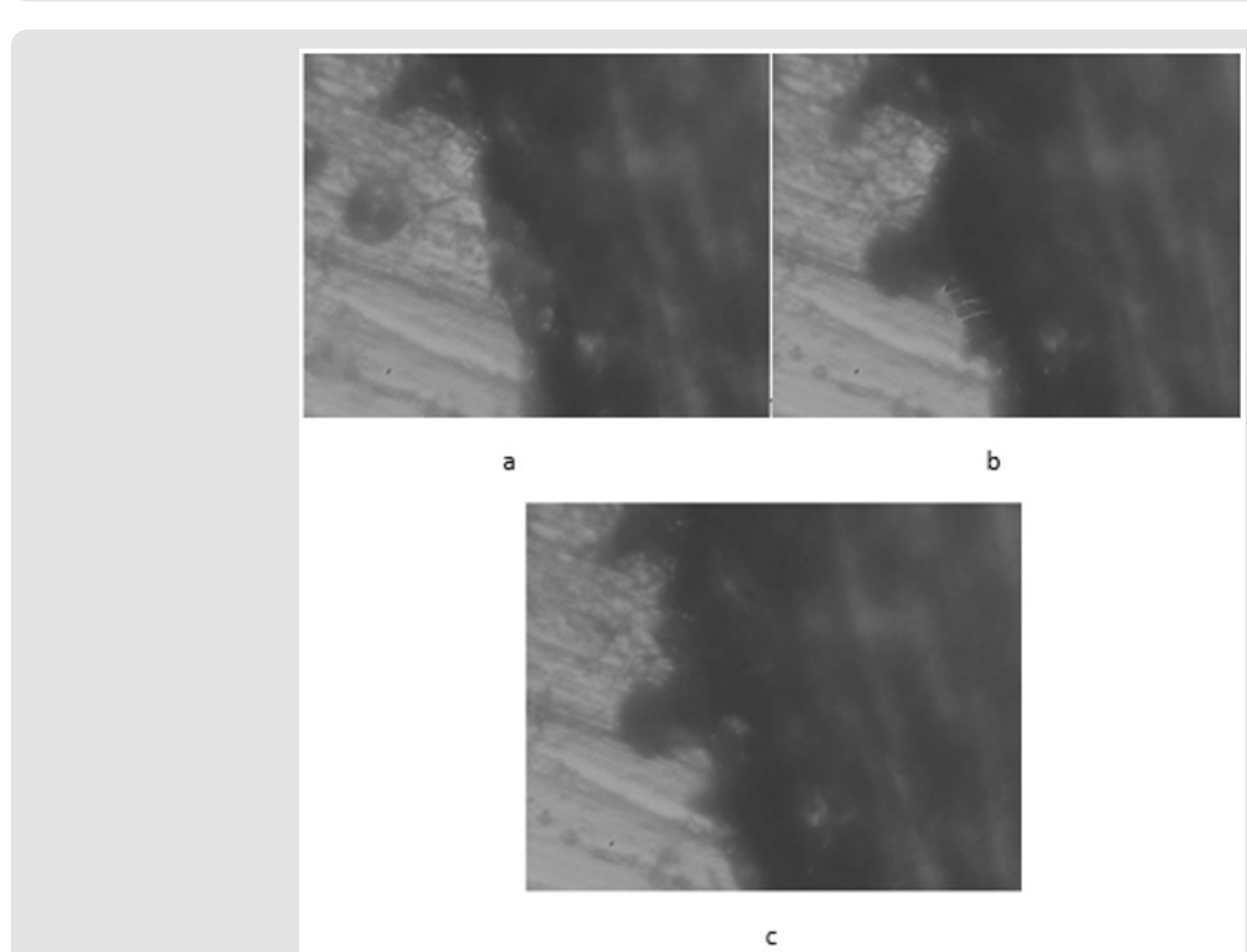

of a "whisker" during the discharge process, when the material of the molten material that has been removed from the hole, the "whiskers" themselves contribute to the subsequent breakdown, where the interelectrode gap is effectively reduced.

The temperature of aluminium and titanium exceeds the temperature of the steel during processing in the same modes. We can explain this phenomenon for titanium thanks to such parameters as increased density and heat capacity, reduced thermal conductivity, which lead to the formation of thermal energy, where contact occurs between the material and the discharge channel. But for aluminium, this phenomenon is not explained due to the fact that this alloy has an increased and reduced heat capacity, which should contribute to heat removal from the processing area. This leads to the idea that the breakdown mechanism of aluminium is significantly different from titanium.

Figure 6: Breakdown in a liquid dielectric. EI - copper, EZ - aluminum a) before the first breakdown; b) after the first breakdown; c) after the second breakdown (increase in 1000). 
After the results of processing steel were obtained and processed, aluminum and titanium, we conducted studies of the hypothesis on a single momentum. The resulting breakdown was carried out between the electrodes with minimal processing, but close to the processing conditions. On Figure 5 presents photographs from a microscope during one breakdown from a variety of obtained on air. The photo shows that there are no formations at the edges of the hole that affect the breakdown process. Studies have been conducted, as a result of which it can be argued that the hole in a liquid dielectric is more important than in air, with the same materials. Figure 6 shows the result of the breakdown of aluminum, where distilled water was used as a dielectric liquid, filamentous structures are visible under the letter B. We believe that these are the very "whiskers" (Figure 6). In our opinion, the "whiskers" depend on the energy of the pulses, when the distance between the electrodes is reduced, and idle pulses are transferred to the working form, depend on the volume of the emerging hole, on the liquid in the interelectrode space, and also on the electrodes after breakdown. But at this point in time, it is not possible to identify the laws of mustache formation, since too many factors influence this formation.

1. It has been experimentally proved that during the processing of steel "whiskers" did not form, since the breakdown does not provoke deviations due to low chemical activity.

2. The possibility of the existence of oxide formations of the "whiskers" of aluminum and titanium is justified, and the justification of the influence of a single breakdown on the entire processing process was also made. The whiskers "during the processing of aluminum formed much more often than during the processing of titanium, since the chemical activity

ISSN: 2574-1241

DOI: 10.26717/BJSTR.2019.21.003597

Sarilov M Yu. Biomed J Sci \& Tech Res

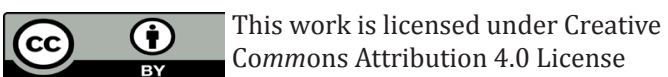

Submission Link: https://biomedres.us/submit-manuscript.php of titanium is lower, although you used the temperature values alone, as well as particles of titanium should be heavier in a material such as titanium, therefore," mustache " are formed less frequently.

3. It is proved that formations of aluminum oxide are obtained of different lengths under different processing conditions. It is proved experimentally that the appearance of oxide formations during the processing of titanium is unlikely.

4. During the experiment, the conditions of thermal phenomena on the electrode blanks of three materials and different modes were identified.

5. A hypothesis was put forward on the formation of filamentary formations after breakdown, which has an effect on the subsequent.

\section{References}

1. M Yu Sarilov, AS Linev (2013) The mechanism of electroerosive breakdown in water for aluminum and titanium alloys Fundamental research. 6(6): 1349-1353.

2. M Yu Sarilov, AS Linev (2013) Investigation of the process of interelectrode breakdown in a liquid dielectric in order to increase the efficiency of electric discharge machining -Scientific notes of Komsomolsk-on-Amur State Technical University. - Komsomolsk-on-Amur: Publishing House KnAGTU 2S: 55-63.

3. M Yu Sarilov, AS Linev (2015) Effect of the electric spark discharge on the efficiency of electric discharge machining of aluminum and titanium alloys - Scientific notes of Komsomolsk-on-Amur State Technical University. - Komsomolsk-on-Amur: Publishing House KnAGTU. 2: 5765.

4. M Yu Sarilov, AS Linev (2016) Improving the efficiency of electrical discharge machining of aluminium and titanium alloys. Journal Metallurgy of mechanical engineering 3: 52-59.

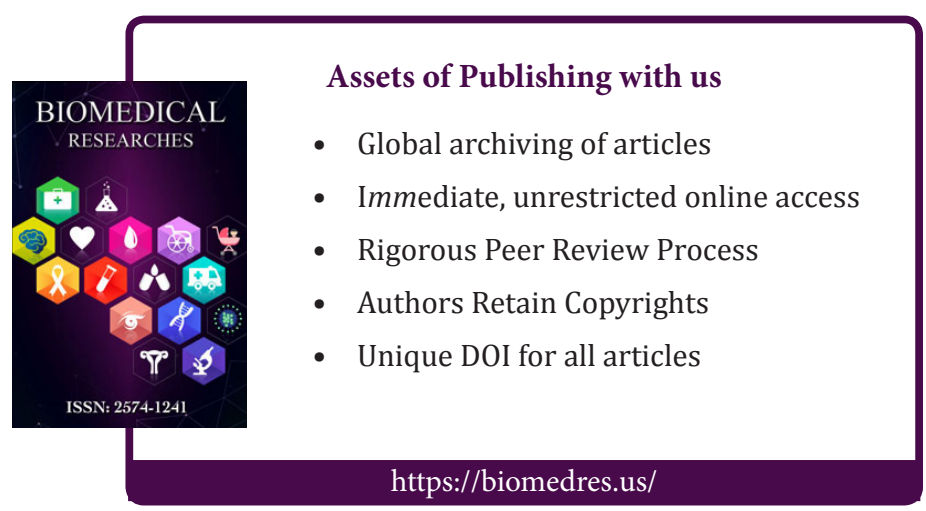

